\title{
Genome-wide analysis of the distribution of AP2/ERF transcription factors reveals duplication and $C B F s$ genes elucidate their potential function in Brassica oleracea
}

Senthil Kumar Thamilarasan, Jong-In Park, Hee-Jeong Jung and III-Sup Nou*

\begin{abstract}
Background: Cabbage (Brassica oleracea) is one of the most important leaf vegetables grown worldwide. The entire cabbage genome sequence and more than fifty thousand proteins have been obtained to date. However a high degree of sequence similarity and conserved genome structure remain between cabbage and Arabidopsis; therefore, Arabidopsis is a viable reference species for comparative genomics studies. Transcription factors (TFs) are important regulators involved in plant development and physiological processes and the AP2/ERF protein family contains transcriptional factors that play a crucial role in plant growth and development, as well as response to biotic and abiotic stress conditions in plants. However, no detailed expression profile of AP2/ERF-like genes is available for B. oleracea.

Results: In the present study, 226 AP2/ERF TFs were identified from B. oleracea based on the available genome sequence. Based on sequence similarity, the AP2/ERF superfamily was classified into five groups (DREB, ERF, AP2, RAV and Soloist) and 15 subgroups. The identification, classification, phylogenetic construction, conserved motifs, chromosome distribution, functional annotation, expression patterns and interaction network were then predicted and analyzed. AP2/ERF transcription factor expression levels exhibited differences in response to varying abiotic stresses based on expressed sequence tags (ESTs). BoCBF1a, 1b, 2, 3 and 4, which were highly conserved in Arabidopsis and $B$. rapa CBF/DREB genes families were well characterized. Expression analysis enabled elucidation of the molecular and genetic level expression patterns of cold tolerance (CT) and susceptible lines (CS) of cabbage and indicated that all BoCBF genes responded to abiotic stresses.
\end{abstract}

Conclusions: Comprehensive analysis of the physiological functions and biological roles of AP2/ERF superfamily genes and BoCBF family genes in B. oleracea is required to fully elucidate AP2/ERF, which will provide rich resources and opportunities to understand abiotic stress tolerance in crops.

Keywords: Brassica oleracea AP2/ERF, Classification, Gene expression, Abiotic stresses, BoCBF genes

\section{Background}

Plant growth, development and yield are influenced greatly by numerous biotic and abiotic stresses, including cold, salt, drought and soil salinization. Plant stresses are generally controlled by a network of specialized genes through intricate regulation by specific transcription factors (TFs). The AP2/ERF superfamily, which is one of the largest groups of TFs families, is involved in the regulation of

\footnotetext{
*Correspondence: nis@sunchon.ac.kr

Department of Horticulture, Sunchon National University, 413 Jungangno, Suncheon, Jeonnam 540-950, Republic of Korea
}

plant developmental processes and biotic and abiotic stress responses $[1,2]$. This family includes all genes encoding at least one APETALA2 (AP2) domain and can be further separated into ETHYLENE RESPONSE FACTOR (ERF), AP2, RAV and Soloist families. The ERF family encodes proteins with a single AP2 domain, while the AP2 gene family codes for TFs with two AP2 domains $[3,4]$. Lastly, the RAV family encodes proteins processing a single AP2 domain plus an additional B3 domain, which is also present in other, non ERF- transcription factors [5]. 
To date, two major efforts to define a nomenclature for ERF sequences have been published. Sakuma et al. [6] divided the Arabidopsis ERF family into two subfamilies based on the amino acid sequence of the DNA binding domain, the CBF/DREB subfamily (group A) and ERF subfamily (group B). These two families were then further subdivided into six groups [7]. Conversely, Nakano et al. [4] divided the AP2/ERF domain amino acid sequences, of Arabidopsis and rice ERF families into 12 and 15 groups, respectively. Similarly, 10 groups were identified in the grape and cucumber ERF family [3].

After release of the whole genomic sequences of several plant organisms, a variety of AP2/ERF TFs were successfully identified and investigated in several plants, including Arabidopsis, rice [4,8], grape [3], poplar (Populus tricocarpa) [9], wheat (Triticum aestivum) [10], cucumbers [5], barley (Hordeum vulgare) [11], and soybean (Glycine max) [12]. The AP2/ERF TFs regulate diverse biological processes in plant function and development, such as hormones, reproduction, cell proliferation, and abiotic and biotic stress responses [13,14].

The DREB subfamily is commonly used as a viable candidate to enhance crop abiotic stress tolerance. The DREB subfamily exhibits different response patterns under environmental stress, including low-temperature (AtCBF1) [15], drought (OsDREB1) [16,17], and high salinity (CaDREBLP1) [18]. The DREB TFs activate multiple dehydration/cold-regulated genes by interacting with DRE/CRT elements (A/GCCGAC), which are present in the Cold response/Responsive to desiccation COR/RD gene promoters [19]. In addition, several DREB subfamily genes are reportedly positive and negative mediators of $\mathrm{ABA}$ and sugar responses, primarily during the germination and early seedling stages [20]. Therefore, the CBF/DREB family genes were annotated and a qRT-PCR platform that allows investigation of their expression profile was developed in the present study.

Cabbage (Brassica oleracea) is one of the most popular vegetable crops worldwide. Currently, Brassica crops are used for human consumption and provide resources for investigation of genome evolution of cabbage [21]. The B. oleracea capitata (line 02-12) genome was recently sequenced and assembled, http://www.ocri-genomics.org/ bolbase/ [22]. Release of the entire cabbage genome sequence, as well as those of Arabidopsis and B. rapa have provided an opportunity for comparative genomic study of AP2/ERF TFs. Characterization of AP2/ERF superfamily genes in $B$. oleracea can clarify the molecular mechanisms responsible for abiotic stress responses to conditions such as cold, salt, drought and ABA, which will enable development of, Brassica varieties with increased tolerance to many adverse environments using transgenic technology. In the present study, 226 putative TFs in the AP2/ERF family were identified from the database of the
B. oleracea genome. Bioinformatic methods were used to analyze the sequence information and construct a phylogenetic tree. Some gene duplication events of AP2/ERF family TFs in $B$. oleracea were found. Identified $B$. oleracea $C B F / D R E B$ genes were then selected for quantitative real-time PCR analysis, to determine the expression level of TFs under different abiotic stresses.

\section{Results and discussion}

Identification of the AP2/ERF family TFs in B. oleracea

The availability of complete $B$. oleracea genome sequences has made it possible to identify all of the AP2/ERF family members in cabbage for the first time. To identify the four family genes, we conducted extensive BLASTN searches based on B. rapa and $A$. thaliana nomenclature suggestions [23,7]. Our extensive search for AP2-domain containing proteins identified 226 distinct AP2/ERF putative TFs (Additional file 1: Table S1). A total of 181 genes with a single AP2/ERF domain were assigned to the ERF family. Additionally, 32 genes were grouped into the AP2 family, based on the presence of a tandem repeated double AP2/ ERF motif. The RAV family included 13 genes identified as encoding one AP2/ERF domain together with one B3 domain. The remaining genes, Bol184 and Bol185 were not only divergent from the ERF family, but were homologous with Arabidopsis and B. rapa Soloist (Br265 and At127, respectively) and therefore designated as Soloist.

Previous annotations of AP2/ERF genes nomenclature proposed by Sakuma et al. [6] were based on homology of the DNA binding domain alone. However, Nakano et al. [4] proposed an alternative method, based on the presence of domains that were different from the DNA binding domain. Therefore, we subdivided the cabbage AP2/ERF genes into 15 groups based on conserved domain similarities to B. rapa and Arabidopsis AP2/ERF TFs. Cumulatively, the number of AP2/ERF TFs in cabbage exceeded that in Arabidopsis (147) poplar (202), and rice (196) [7]. The proportion of each subfamily is shown in Table 1 and Additional file 2: Figure S1. The RAV family number in cabbage (13) was larger than that in other plant groups, including Arabidopsis (6), rice (4), and tomato (3), but lesser than that of $B$. rapa (14). Cabbage contained more AP2 double domain genes in each subgroup than Arabidopsis, and the DREB A-4 subgroup had up to two times more than Arabidopsis.

\section{Phylogenetic analysis of AP2/ERF TFs family}

To investigate the evolutionary relatedness of the identified sequences together with AP2/ERF genes encoded by the other fully sequenced Chinese cabbage and Arabidopsis, we performed phylogenetic reconstruction using the conserved AP2/ERF transcription factor domain. The resulting phylogenetic tree (Figure 1) resolved 15 clades containing the ERF, AP2, RAV and Soloist families, which was 


\begin{tabular}{|c|c|c|c|c|}
\hline \multicolumn{2}{|l|}{ Plant } & \multirow{2}{*}{$\begin{array}{l}\text { Brassica } \\
\text { oleracea } \\
\text { Number }\end{array}$} & \multirow{2}{*}{$\begin{array}{l}\text { Arabidopsis } \\
\text { thaliana } \\
\text { Number }\end{array}$} & \multirow{2}{*}{$\begin{array}{l}\text { B. rapa subsp. } \\
\text { Pekinensis } \\
\text { Number }\end{array}$} \\
\hline Classification & Group & & & \\
\hline \multirow[t]{7}{*}{ DREB subfamily } & $\mathrm{A} 1$ & 8 & 6 & 10 \\
\hline & $\mathrm{A} 2$ & 9 & 8 & 20 \\
\hline & A3 & 1 & 1 & 5 \\
\hline & A4 & 33 & 16 & 35 \\
\hline & A5 & 23 & 16 & 27 \\
\hline & A6 & 17 & 10 & 15 \\
\hline & Total & 91 & 57 & 112 \\
\hline \multirow[t]{7}{*}{ ERF subfamily } & $\mathrm{B} 1$ & 19 & 15 & 22 \\
\hline & B2 & 10 & 5 & 10 \\
\hline & B3 & 25 & 18 & 35 \\
\hline & B4 & 5 & 7 & 14 \\
\hline & B5 & 19 & 8 & 28 \\
\hline & B6 & 10 & 12 & 23 \\
\hline & Total & 88 & 65 & 132 \\
\hline \multicolumn{2}{|l|}{ AP2 subfamily } & 32 & 18 & 30 \\
\hline \multicolumn{2}{|l|}{ RAV subfamily } & 13 & 6 & 14 \\
\hline \multicolumn{2}{|l|}{ Soloist } & 2 & 1 & 1 \\
\hline \multicolumn{2}{|c|}{ Total AP2/ERF family factors } & 226 & 147 & 289 \\
\hline
\end{tabular}

in accordance with previous studies [7,23]. Groups I to VI represent the DREB subfamily, while groups VII to XII represent the ERF subfamily and groups XIII, XIV and XV indicate the AP2, RAV and Soloist families, respectively. Although the Soloist transcription factor contained a single AP2 domain in cabbage, it clustered with the RAV family, while the Soloist transcription factor grouped with the AP2 family in grape [3]. We conducted a more in depth phylogenetic analysis of the AP2 family by selecting the AP2 family proteins, which contained two AP2 domains. Chinese cabbage and Arabidopsis were divided into two groups, AP2-R1 and AP2-R2, respectively (Additional file 2: Figure S2).

The conservative motifs among AP2/ERF proteins in plants were clarified by conducting multiple alignment analyses using amino acid sequences of the AP2/ERF domain. Several genes were selected from each species of the AP2/ERF family to identify the motifs. Sequence alignment showed that the motif length in the RAV and Soloist family was longest, and contained 50 amino acids, followed by DREB (41 amino acids). However, the AP2 family contained two groups (AP2-R1 and AP2-R2), that might be responsible for the reduced motif length (29 amino acids). The divergence between the two groups might affect the AP2 family motif length, with a higher divergence generally being associated with a shorter motif. The same explanation might be used for the ERF subfamily (26 amino acids), which contained six groups, and a shorter conserved motif. Although six groups were identified in the DREB and ERF subfamilies, the conserved motif was much longer than in the ERF subfamily (Additional file 2: Figure S3). The differences within the AP2/ERF family were further analyzed by examining the DREB, ERF and AP2 conserved motifs using the MEME program. The results showed that all but two (DREB-A2 and DREB-A6) of the six groups had a 50 amino acid conserved motif, which contained 32 and 29 amino acids in the conserved motif, respectively (Additional file 2: Figure S4). In the ERF subfamily, a shorter conserved motif was observed in ERF-B2, B3, $\mathrm{B} 5$ and $\mathrm{B} 6$ than in the remaining ERF group (50 amino acids). Therefore, if the ERF subfamily motif length was dependent on the ERF-B5 group, it might be responsible for the overall shorter ERF subfamily motif. The AP2-R2 group contained 29 amino acids identified in the AP2-R1 group conserved motif (Additional file 2: Figure S5 and Figure S6).

Sequence alignment of all AP2/ERF families indicated that LG, AA and YD elements were highly conserved (Additional file 2: Figure S3). The WLG element in DREB, ERF and RAV was more highly conserved than that in AP2 and Soloist. In the Soloist family, some WLG elements were converted into HLG elements. The AYD element was conserved in the AP2/ERF superfamily, with the exception of Soloist, where it was converted into LYD (Additional file 2: Figure S3). We assume that specific conserved motifs are related to molecular functions. We use this assumption as an effective and practical means to predict unknown protein functions were derived from structural relationship in Arabidopsis [4].

\section{Chromosome distribution of the AP2/ERF TFs family}

Among all AP2/ERF family TFs resolved in the cabbage genome, 91 genes belong to the DREB subfamily, followed by 88 in ERF, 32 in AP2, 13 in RAV and two belonging to Soloist (Table 1 and Additional file 2: Figure S1). A total of $185 \mathrm{AP2} / \mathrm{ERF}$ TFs were distributed on nine cabbage chromosomes (Figure 2 and Additional file 1: Table S3), and while 41 genes could not be assigned to any specific chromosome. Chromosome 6 and 7 had the highest number of AP2/ERF TFs (26 and 27, respectively), while the lowest AP2/ERF transcription factor number was found on chromosome 5 (13 genes) and 1 (15 genes). The high AP2/ERF sequence number on chromosomes 6 and 7 was primarily due to the increased number of DREB (10 and 9) and ERF (13 and 9) subfamilies. Interestingly, conserved sequences and the physical proximity of repetitive TFs, that belonged to the same group were identified and located on the same chromosomal regions as follows: Bol010 to Bol011 and Bol018 to Bol019 (XV group) were located on chromosome 2; Bol116 to Bol117 (II group), 


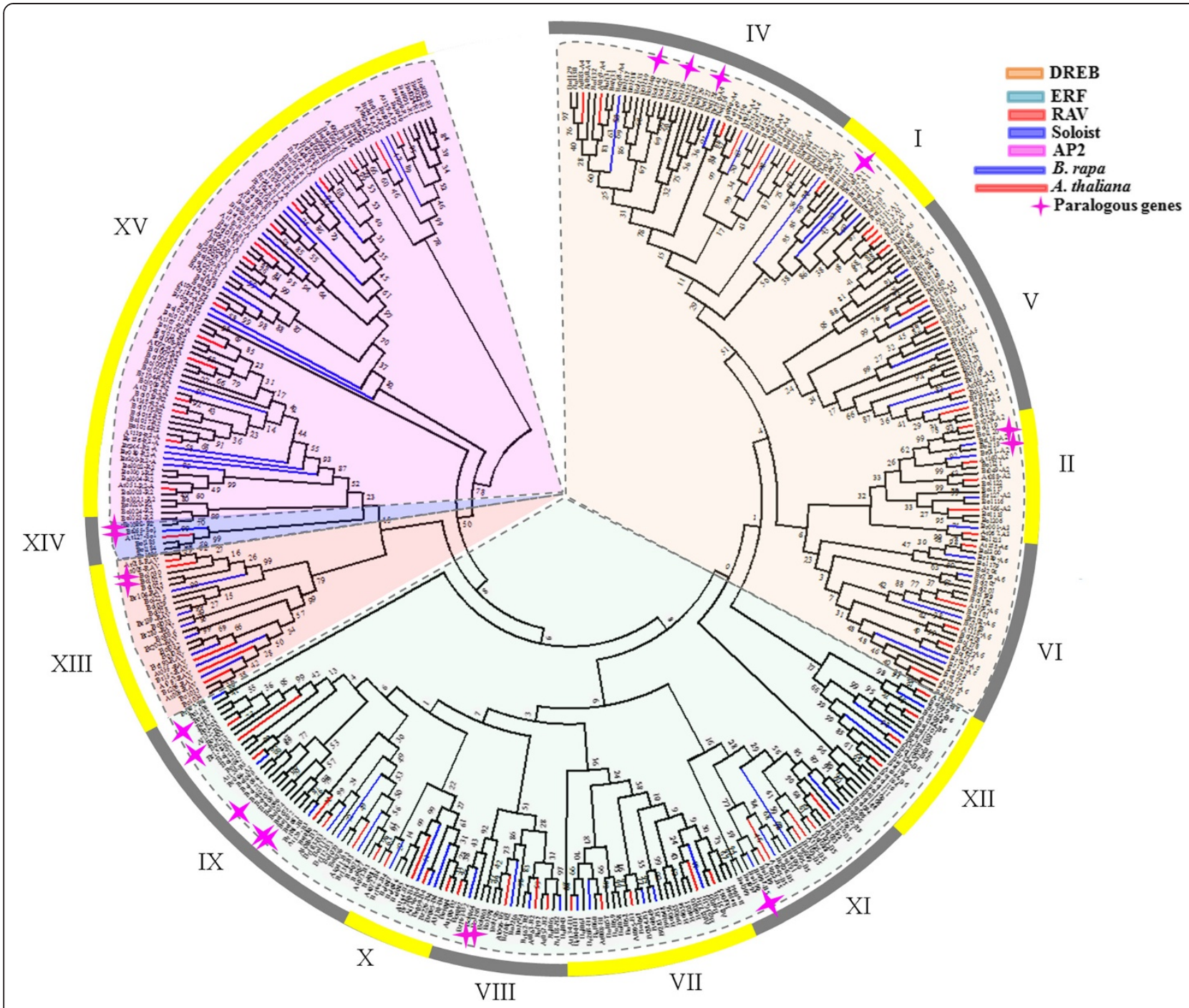

Figure 1 Phylogenetic tree constructed by the neighbor-joining method using AP2/ERF transcription factor domains in cabbage, Chinese cabbage and Arabidopsis. The tree was divided into 15 groups that contained ERF (DREB and ERF subfamily), AP2, RAV and the Soloist family. The star represents paralogous genes of cabbage. Lines represent Chinese cabbage (blue) and Arabidposis (red).

Bol124 to Bol125 (IV group) and Bol210 to Bol211 (IX group) were located on chromosome 4; Bol118 to Bol119 (II group), Bol135 to Bol136 (IV group), Bol157 to Bol158 (V group) and BolO27 to BolO30 (XIII group) were located on chromosome 7; Bol056 to Bol057 (VII group) and Bol093 to Bol094 (XI) were located on chromosome 6; Bol182 to 185 (VI, XIV) and Bol104 to Bol105 (XII) were located on chromosome 8; Bol073 to Bol74 (IX group) were located on chromosome 9 (highlighted in Additional file 1: Table S1). Similar patterns were also found in the Arabidopsis [6], B. rapa [23], grape and poplar genomes $[3,10]$, which were suggested to represent paralogous segments resulting from ancestral polyploidization events. The highest number of RAV TFs was found on chromosome 6 (4 genes), followed by chromosomes 2 , $3,5,8$ and Cun $(2,1,1,1$ and 4 genes respectively), while
RAV TFs were not detected on chromosomes 1, 4, 7 and 9. Moreover, 17.5 and $19.3 \%$ of the DREB and ERF TFs were observed on chromosome scaffolds (Additional file 1: Table S3).

We also investigated the cabbage paralogous AP2/ERF TFs. A total of 198 AP2/ERF TFs showed high homology (>85\%) to the AP2/ERF genes (Additional file 1: Table S4). The paralogous genes were indicated in the phylogenetic tree (Figure 1). Duplication events have been studied in grape, and 17 proteins with high similarity sequences were reported [3]. In the present study, we identified 14 genes in the cabbage genome with $100 \%$ sequence similarity. Among the 14 duplicated genes, eight were ERF-subfamily members, while the rest belong to the DREB subfamily (4 genes) and RAV subfamily (2 genes) (Additional file 1: Table S4). 


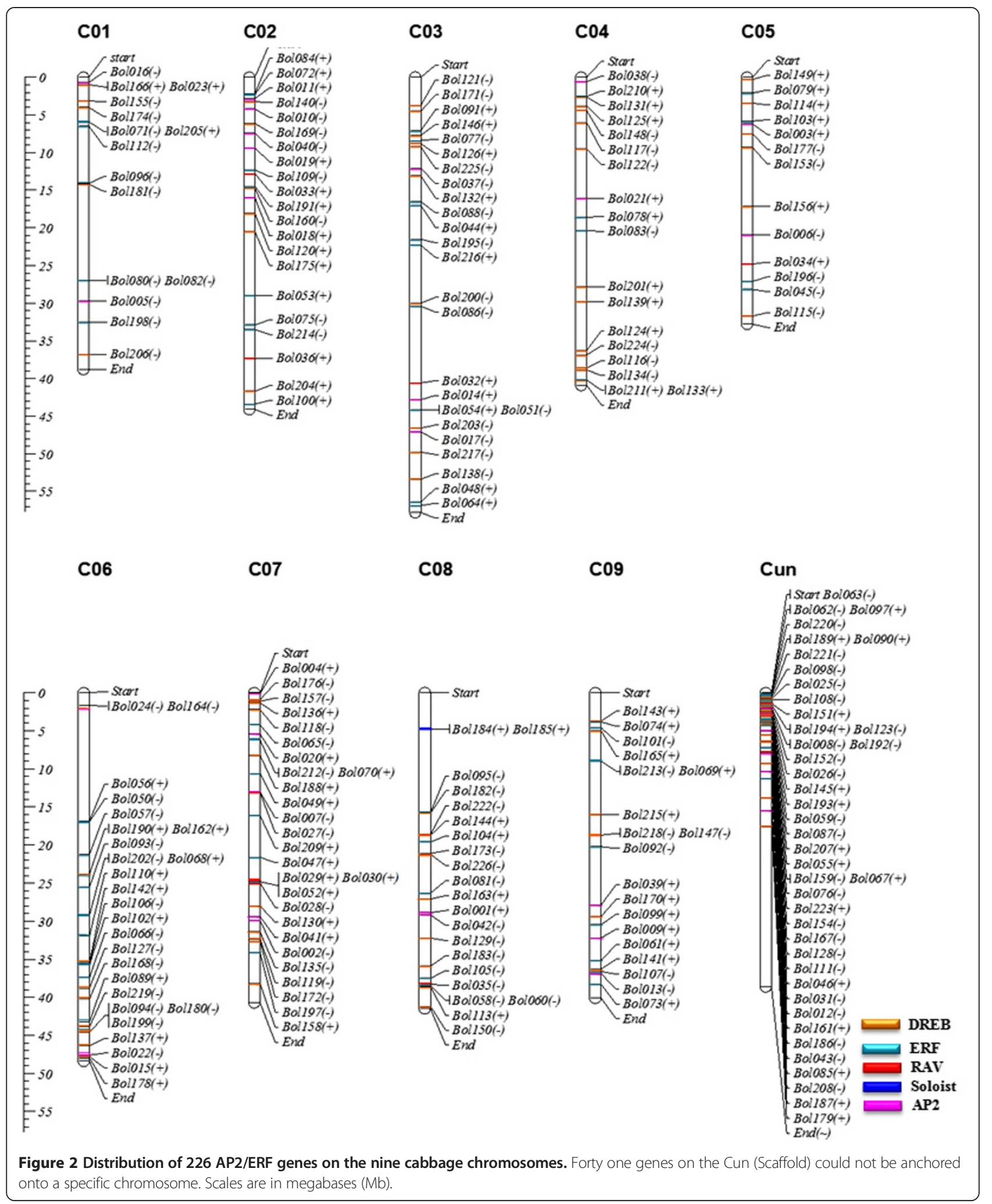

AP2/ERF TFs expression patterns in $B$. oleracea

We also investigated the expression patterns of $B$. oleracea AP2/ERF genes during abiotic stress condition, such as cold, salt and drought using the microarray data. In this study, based on Arabidopsis BLAST hits, cold, salt and drought expression profiles were downloaded from the 
AtGenExpress visualization tool to evaluate responses to stress [24]. The results provided rich resource for gene discovery and investigation of gene expression patterns. A total of 221 AP2/ERF TFs were obtained by expression profile tags (Additional file 1: Table S5). Most AP2/ERF proteins belonged to the DREB and ERF subfamily. In the DREB family, gene relative expression profiles exhibited in all time courses had a high degree of expression pattern such as up (red) and down (green) - regulated genes. A few genes were not expressed (black) in response to any stress or throughout the study period (Figure 3). Detailed expression values and clusters of each AP2/ERF family transcription factor were analyzed using cluster analysis based on Arabidopsis best hits of gene expression value (Additional file 2: Figure S7- S9). Conversely, the predicted B. oleracea AP2/ERF genes were annotated based on sequence alignment to non-redundant (nr) public databases using BLASTN. Each AP2/ERF was annotated from the $\mathrm{nr}$ database was integrated and results provided in a supplemental file (Additional file 1: Table S6). Interestingly, we identified five CBF proteins with the highest similarity to Arabidopsis protein might be related to cold and freezing tolerance in $B$. oleracea from Bolbase database using BLASTN. Cellular localization is often an important factor in determining protein function. Liu et al. [25] has suggested that TFs are located only in the nucleus. PSORT was applied to predict and determine localization of BoCBF proteins was in the nucleus. Gene structure revealed that the all $B o C B F s$ had almost no introns. It was one of the representative characteristics of the CBFs family members, CBF proteins were well characterized listed in the Table 2, Additional file 2: Figure S10 [26], and all five of the aforementioned genes were identified as the members of the DREB-A1 group. Subsequently, cabbage and Arabidopsis protein interactions, including functional and physical interactions were examined using the STRING software and the corresponding database to identify the protein interactions [27]. Five proteins that exhibited increased sequence similarity to CBF1 (Bol217, Bol220) and CBF2 (Bol219) were involved in stronger (thicker lines)

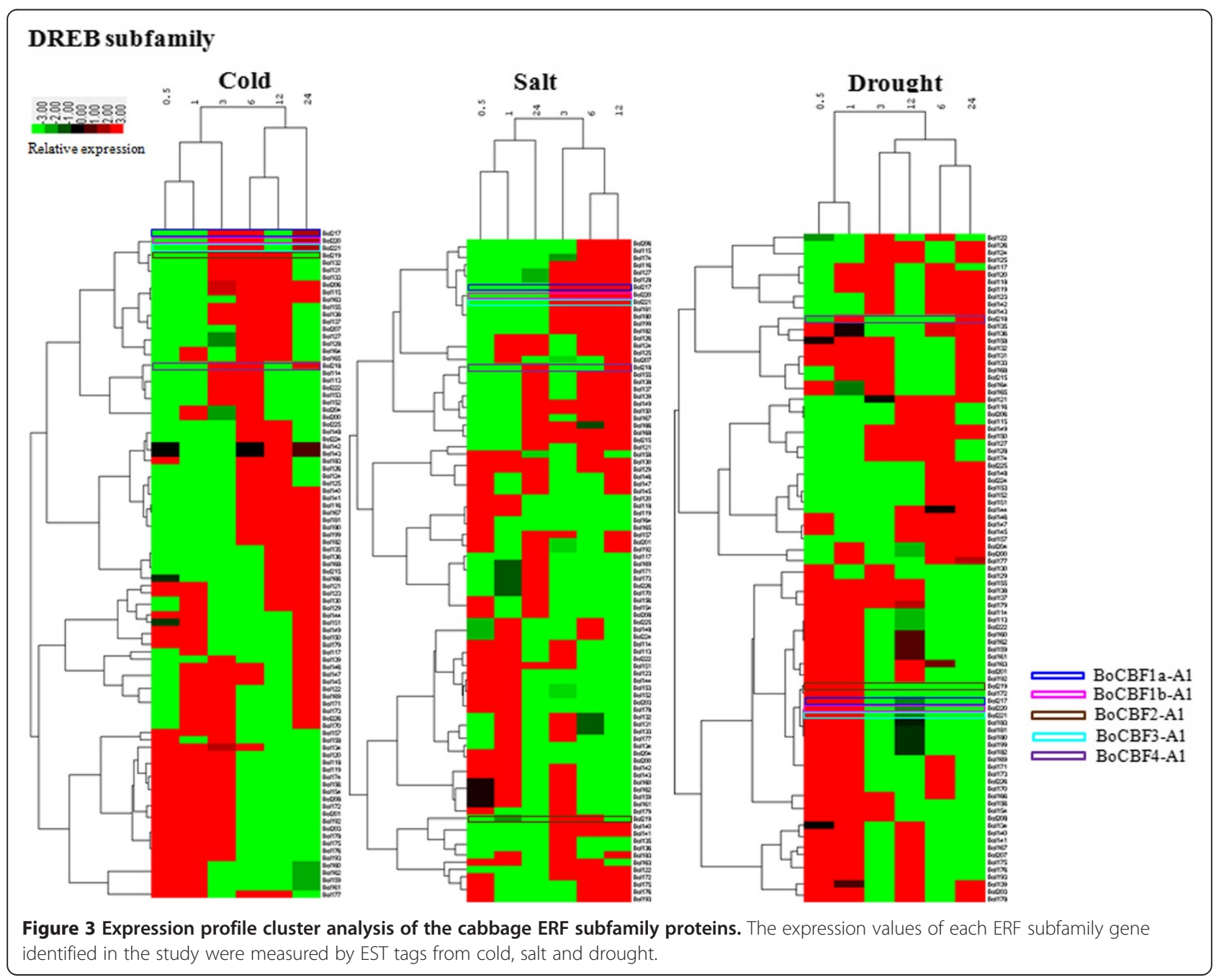


Table 2 Amino acid sequence characteristics of BoCBF genes

\begin{tabular}{|c|c|c|c|c|c|c|c|c|c|c|c|c|}
\hline \multirow[t]{2}{*}{ Gene ID } & \multirow{2}{*}{$\begin{array}{c}\text { Sequenced ID } \\
\text { (Bo/base) }\end{array}$} & \multirow{2}{*}{$\begin{array}{l}\text { TAIR homology } \\
\text { (aa) (\%ld) }\end{array}$} & \multirow{2}{*}{$\begin{array}{l}\mathrm{Nt}(\mathrm{bp}) \\
\text { length }\end{array}$} & \multirow[t]{2}{*}{ Chr. no., position } & \multirow[t]{2}{*}{ aa length } & \multirow[t]{2}{*}{$\mathrm{pl}$} & \multirow[t]{2}{*}{ Mol. wt(kDa) } & \multirow[t]{2}{*}{ a-helix (\%) } & \multirow[t]{2}{*}{$\beta$ - sheet (\%) } & \multicolumn{2}{|c|}{ NLS } & \multirow{2}{*}{$\begin{array}{l}\text { Subcellular } \\
\text { localization }\end{array}$} \\
\hline & & & & & & & & & & Monopartite & Bipartite & \\
\hline BoCBF1a & Bol029967 & AT4G25490 (68) & 645 & C03; 49752542-49753186 (-) & 214 & 5.03 & 23.77 & 32.71 & 7.94 & $65-79$ & - & $n / 13.0$ \\
\hline BoCBF1b & Bol006459 & AT4G25490 (59) & 753 & Cun; 000278: 15959-16711 (-) & 250 & 4.85 & 27.86 & 30.80 & 11.20 & $64-78$ & $33-60,196-228$ & $n / 12.0$ \\
\hline BOCBF2 & Bol042239 & AT4G25470 (62) & 648 & C07; 43885424-43886071 (-) & 215 & 5.34 & 23.82 & 30.23 & 10.23 & $66-80$ & - & $n / 11.0$ \\
\hline BOCBF3 & Bol006460 & AT4G25480 (50) & 834 & Cun; 000278: 49871-50704 & 277 & 4.58 & 30.45 & 36.10 & 9.75 & $68-82$ & $224-255$ & $n / 10.0$ \\
\hline BOCBF4 & Bol029422 & AT5G51990 (68) & 663 & C09; 18733153-18732491 & 220 & 6.14 & 24.51 & 35.45 & 9.09 & $40-49,69-83$ & - & $n / 12.0$ \\
\hline
\end{tabular}

aa- amino acid; NLS- nuclear localization signal; $n$ - nucleus. 
interaction network. The Bol218 protein, which showed high homology with CBF4, was involved in weak interactions (thinner lines) with all genes (Figure 4). The former network largely participated in cold regulatory pathways, as most factors were related to cold stress, including COR47, COR15A, KIN2, CBF1, CBF2, and ADA2A [28]. Many homologues are known to be induced rapidly upon exposure to low temperature, such as the AtCBF1-3 genes from Arabidopsis [29], and the ZmDREB1A gene from Zea mays [30]. AtCBF4 is not induced by cold, but the CBF4 network might be involved in drought adaptation [31,32].

There were a large number of DREB- and ERF-type sequences related to stress. We obtained five $B o C B F$ family genes based on the Arabidopsis protein interaction. Quantitative real-time PCR was used to analyze the expression profile of $C B F / D R E B$ family genes under cold, salt, drought and ABA stresses using two contrasting genotype lines: cold tolerant Bo106 (CT) and cold susceptible $\mathrm{Bo107}$ (CS). All genes analyzed exhibited differential accumulation in response to cold, salt, drought and ABA (Figure 5, Additional file 3).

Evaluation of $C B F$ gene expression revealed that some $C B F$ genes in plant species could be induced by short term exposure to cold stress [33]. In our study, five novel $B o C B F$ genes showed different expression patterns when the plants were exposed to cold stress. Detected transcripts, $B o C B F 2$ and $B o C B F 4$ were negatively regulated by

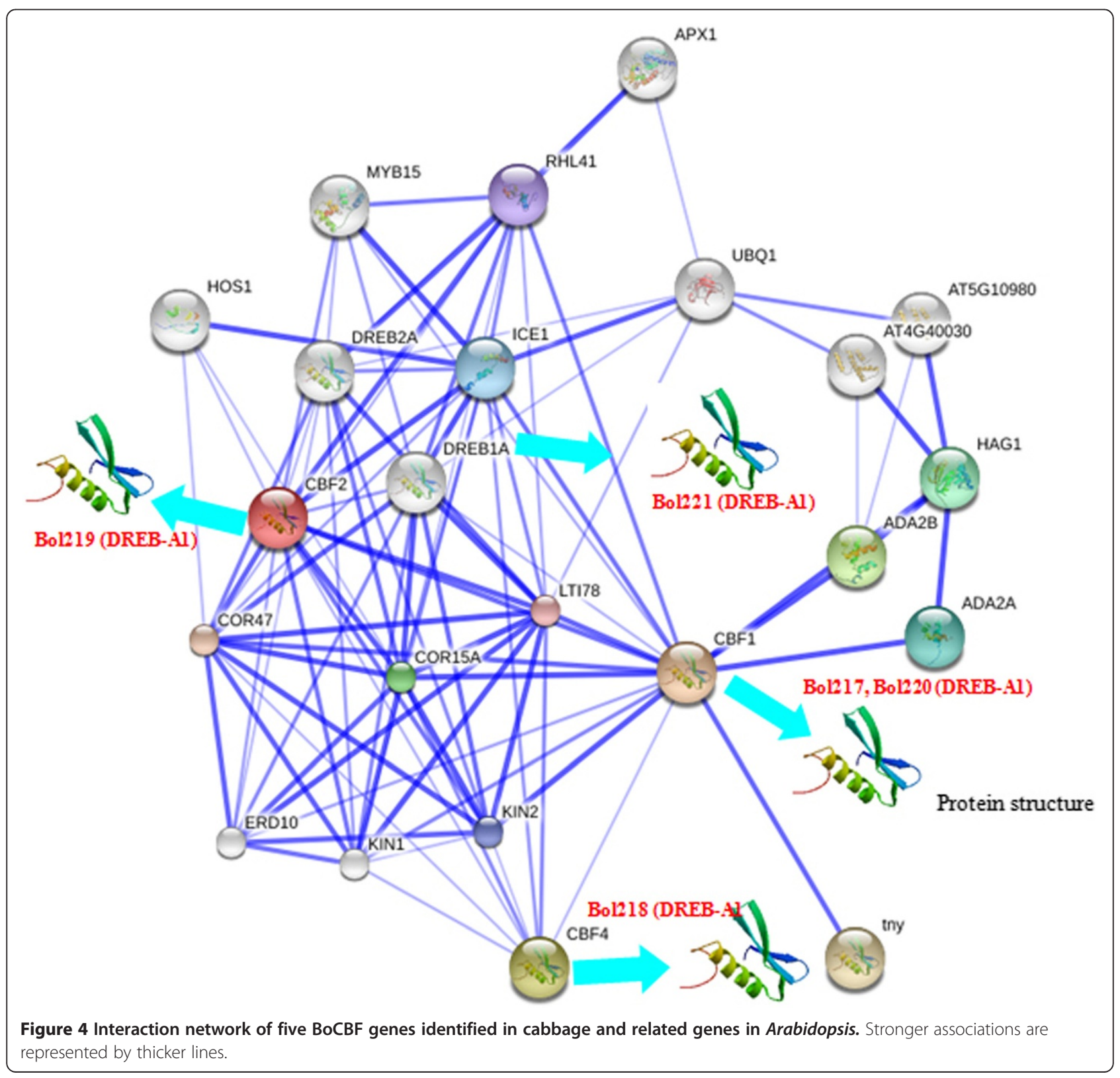




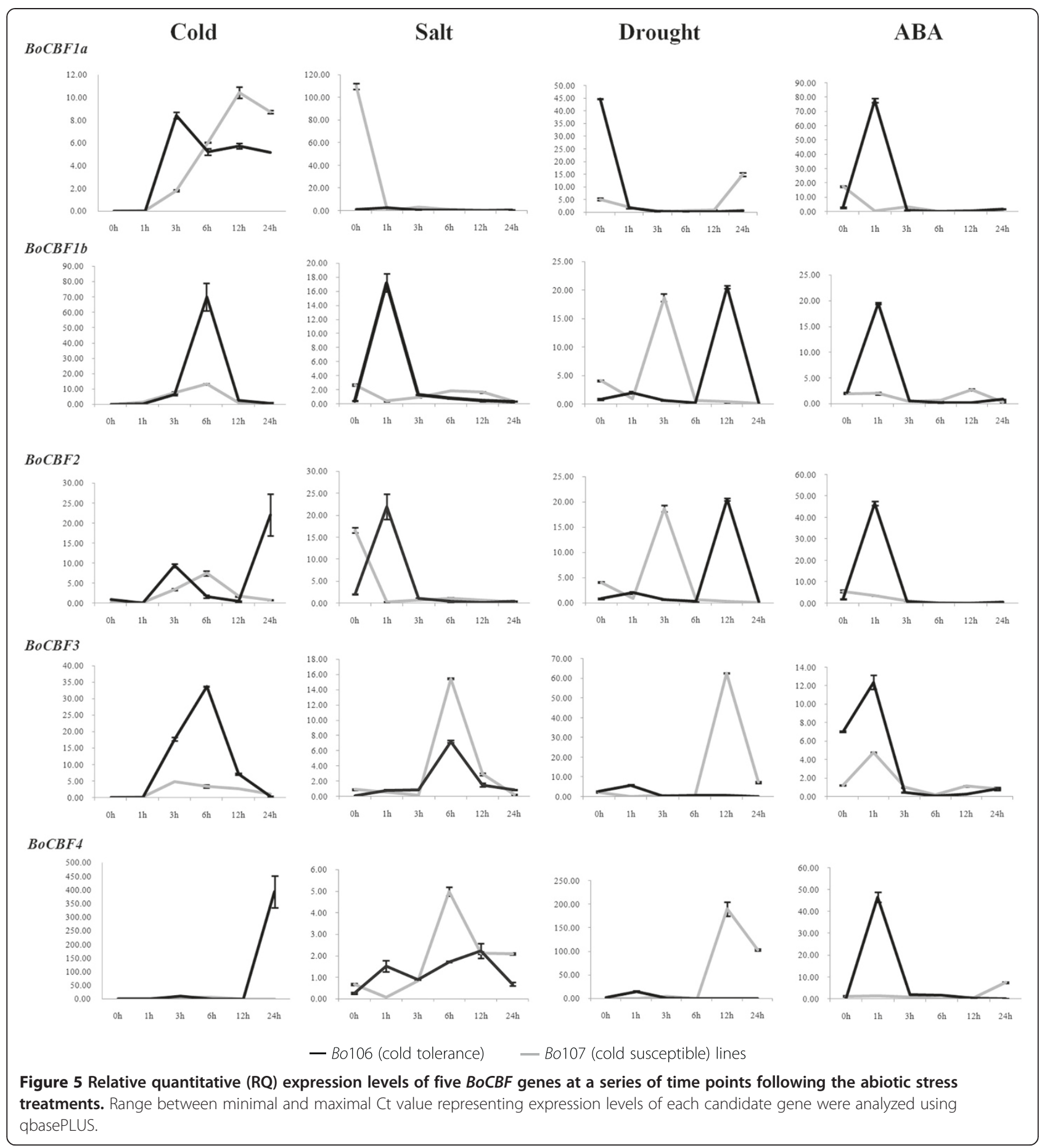

cold stress; however, the patterns among them were not the same. Specifically, BoCBF4 was more highly up-regulated at $24 \mathrm{~h}$ than $B o C B F 2$, while $B o C B F 2$ was elevated at $3 \mathrm{~h}$, then declined after $6 \mathrm{~h}$, but was maintained at a level higher at 24 h. $B o C B F 1 b$ and $B o C B F 3$ were also negatively regulated by cold stress. BoCBF1b was more highly elevated at $6 \mathrm{~h}$ than BoCBF3. However, BoCBF1b and BoCBF3 had a similar expression pattern after $6 \mathrm{~h}$, which might be resistant to cold stress. BoCBF1a was regulated by both positive and negative at $3 \mathrm{~h}$. CT line was more highly regulated at $3 \mathrm{~h}$ than $\mathrm{CS}$, but was maintained at a level higher at $12 \mathrm{~h}$. Overall, these findings indicate that $B o C B F$ genes might be involved in mediating cold signaling transduction when plants are exposed to cold temperature. However, Haake et al. [33] and Medina et al. [26] reported that $C B F$ gene expression was modulated by 
salt and dehydration. $B o C B F 3$ and $B o C B F 4$ were positively regulated by salt stress; however, they showed similar expression patterns and maintained the high abundance at 6 h. BoCBF1b and BoCBF2 had similar expression pattern were negatively regulated by salt stress, which are shortly induced at $1 \mathrm{~h}$ followed by gradual decrease after $3 \mathrm{~h}$ which might be responsible resistant to salt stress. It should be noted that $B o C B F$ genes showed stress responsive (positive or negative) at $1 \mathrm{~h}$, indicating that they play an important role in mediating salt signal transduction when plants are exposed to salt stress at short time. $B o C B F 1 b$ and $B o C B F 2$ had differential expression pattern, however, they had similar expression. CS and CT line has maintained the high abundance at $3 \mathrm{~h}$ and $12 \mathrm{~h}$ in both the genes respectively. $B o C B F 1 a, B o C B F 3$ and $B o C B F 4$ were positively regulated by drought stress. However, these genes were not the same responses as at cold and salt stress. BoCBF3 and $B o C B F 4$ were gradually elevated at $12 \mathrm{~h}$, after which it declined. These results indicate that $B o C B F$ genes were dramatically influenced by drought stress, indicating, that they are involved in drought signal transduction through a positive regulation pathway. Hong et al. [34] and Kirch et al. [35] suggested that most cold-regulated genes are also responsive to ABA. In contrast, Medina et al. [26] reported, that $C B F$ transcripts did not accumulate in response to $\mathrm{ABA}$. Hence, it is necessary to determine whether the accumulation of CBF genes was specifically regulated by cold, salt and drought or was also influenced by ABA stress. Here, all $B o C B F$ genes were shortly induced and negatively regulated by ABA stress. BoCBF3 was showed similar expression pattern between $\mathrm{CT}$ and $\mathrm{CS}$ lines, which may not be response to ABA stress. $B o C B F 3$ also showed a similar expression pattern between the $\mathrm{CT}$ and $\mathrm{CS}$ lines, although $\mathrm{ABA}$ resistance gradually decreased than relative to the $C T$ line. These findings indicate that $B o C B F 1 a, B o C B F 1 b, B o C B F 2$ and $B o C B F 4$ transcripts were negatively regulated in the ABA signaling pathway. On the other hand, Andersen et al. [36] was suggested that, estimate the overall expression variation of the candidate genes and variation between the samples subgroups of the sample set. The candidates with lowest intergroup and intragroup variations give the lowest stability $(S)$ value and are therefore ranked higher as more stable. We identified as the most stable expression in cold BoCBF3_S (0.010), salt BoCBF1b_S (0.029), drought BoCBF2_S (0.043) and ABA BoCBF3_S (0.010) (detailed see in Additional file 3). This data indicates significantly more reliable qRT-PCR analysis. Therefore, some of the novel BoCBF genes identified in our study could possibly be used as target genes to improve plant resistance to abiotic stresses such as cold, salt, drought and ABA. It will be useful to evaluate the functions of the $B o C B F$ genes, to generate transgenic crops with tolerance to abiotic stresses in the future.

\section{Conclusions}

In summary, we identified $226 \mathrm{AP} 2 / \mathrm{ERF} \mathrm{TFs}$ in the cabbage genome and characterized their expression patterns in response to different abiotic stresses. To date, this is the first comprehensive and systematic investigation of cabbage AP2/ERF TFs. We conducted quantitative RT-PCR analysis of some of the novel B. oleracea $C B F$ genes identified in this study and found that they have the potential for use as target genes to improve plant resistance to abiotic stresses, such as cold, drought, salt and $\mathrm{ABA}$ in two contrasting genotypes of cabbage. It will be useful to evaluate the functions of the $B o C B F$ genes to generate transgenic crops tolerant of abiotic stresses. Overall, our genomic and bioinformatic analysis of the five family TFs and proteins presented in this work will provide an important foundation for further functional evaluations under different stress conditions.

\section{Methods \\ AP2/ERF superfamily transcription factor identification}

A search of the B. oleracea genome database [22] was conducted to identify all members of the AP2/ERF family. A double strategy to obtain every gene of the AP2/ERF family in the genome was used. The genome DNA database in B. rapa subsp. Pekinensis was downloaded from the Brassica database website (http://brassicadb.org/brad/) $[37,38]$. The sequences of all AP2/ERF superfamily members in the genome of other species assessed were downloaded from the plant TFDB database (http://planttfdb. cbi.edu.cn/) $[39,40]$ and the amino acid sequence of one or most representative members (i.e. the maximum number of different conserved motifs distinctive of each group) for each group defined by Nakano et al. [4] were used as queries to search the Bolbase database. Every sequence identified was subsequently checked against the Arabidopsis and $B$. rapa databases to confirm that it belonged to the AP2/ERF superfamily. As a final quality check, we confirmed the presence of the AP2 domain in every AP2/ ERF B. oleracea gene candidate using SMART [41]. Each subfamily motif was identified using the MEME program [42]. The physical distribution of AP2/ERF genes on chromosomes was drawn by MapChart based on gene position in the genome [43].

\section{Phylogenetic tree construction}

Phylogenetic and molecular evolutionary analyses were conducted using MEGA5.1 [44]. The retrieved conserved domains of AP2/ERF proteins were used to construct phylogenetic trees. The neighbor-joining method was applied to construct different AP2/ERF transcription factor domain trees, using the pair-wise deletion option. 
Tree reliability was assessed using 1000 bootstrap replicates and the numbers indicated for each clade represent bootstrap support values given as percentages. The data matrix and resulting trees were deposited in TreeBASE (accession S15847, http://purl.org/phylo/treebase/phylows/ study/TB2:S15847? $x$-access - code $=171776387$ c9f3d2134e 04184bd5277dc\&format $=$ html).

\section{AP2/ERF superfamily transcription factor expression patterns in B. oleracea}

B. oleracea AP2/ERF superfamily CDS (coding domain sequence) was used to search against the TAIR database using the BLAST tool. The eligible hits (E-value $<1 \mathrm{e}-5$, Identity $>90 \%$ ) were selected for each $B$. oleracea AP2/ERF superfamily transcription factor. Based on Arabidopsis eligible hits, cold, salt and drought expression data were downloaded from the AtGenExpress visualization tool (AVT) (http://jsp.weigelworld.org/expviz/expviz.jsp). The AP2/ERF protein expression cluster from each stress was analyzed via the Cluster program (http://bonsai.hgc. $\mathrm{jp} / \sim$ mdehoon/software/cluster/software.htm), and results were shown using the Tree View software (http://jtreeview. sourceforge.net/).

\section{Identification of AP2/ERF annotation and specific gene interaction networks}

Cabbage AP2/ERF genes were used as a query to search against available public databases (e-value 1.0E-3) using Blast2go [45], which is a sequence-based tool to assign GO terms and annotation for each BLAST hit obtained by mapping the extant annotation associations [46]. The GO terms for each of the three main categories (biological process, molecular function and cellular component) were obtained from sequence similarity using the default parameters. From these annotations, the $2^{\text {nd }}$ Group level and multilevel GO terms were based on the biological process, molecular function and cellular components. This annotation was simplified and focused on plant related functional categories using Plant GOslim. Specific protein interactions were constructed by applying the STRING software (Search Tool for the Retrieval of Interacting Genes/Proteins, http://string-db.org/) [27].

\section{Plant materials, abiotic stress conditions}

For the expression study, Bo106 and Bo107 cold tolerance (CT) and susceptible lines (CS) of cabbage seeds were grown aseptically on half-strength MS agar medium in a culture room under a $16 \mathrm{~h}$ light photoperiod at $25^{\circ} \mathrm{C}$. After three weeks of growth, the seedlings were transferred to fresh liquid MSH (half-strength MS medium without sucrose) medium containing $250 \mathrm{mM} \mathrm{NaCl}$ and $100 \mathrm{mM}$ abscisic acid (ABA) for $24 \mathrm{~h}$. To induce cold stress, the seedlings were maintained at $4^{\circ} \mathrm{C}$ for $24 \mathrm{~h}$. Drought treatment was then applied by keeping the seedlings on the filter paper at $28^{\circ} \mathrm{C}$ for 24 . The samples were then subjected to all stresses for 0 (wild type), 1, 3, 6, 12 and $24 \mathrm{~h}$. Next, the samples were frozen immediately in liquid nitrogen and stored at $-80^{\circ} \mathrm{C}$ until RNA isolation. The total RNA was extracted from the frozen samples of the roots, stems, leaves and flower buds of healthy plants and those exposed to abiotic stress using an RNeasy mini kit (Qiagen, USA), after which RNA was treated with RNase-free DNase (Promega, USA) to remove genomic DNA contamination.

\section{RT- PCR and real time PCR analyses}

RT-PCR was performed using an Avian Myeloblastosis Virus (AMV) one step RT-PCR kit (Takara, Japan). The specific primers for the $B o C B F$ genes are listed in the Additional file 1: Table S7. RT-PCR was performed using $50 \mathrm{ng}$ cDNA of plants exposed to various abiotic stresses, including cold, salt drought and ABA for 0, 1, 3, 6, 12 and $24 \mathrm{~h}$. Next, PCR samples with a total volume of $20 \mu \mathrm{l}$ containing $1 \mu \mathrm{l}$ of first-strand cDNAs and $2 \mu \mathrm{l}$ of primers were subjected to 30 cycles of $30 \mathrm{~s}$ denaturing at $94^{\circ} \mathrm{C}$, annealing at $58^{\circ} \mathrm{C}$ for $30 \mathrm{~s}$ and extension at $72^{\circ} \mathrm{C}$ for $45 \mathrm{~s}$. The PCR products were electrophoresed on a $2 \%$ agarose gel, after which real-time PCR was performed on an Illumina Eco real time machine (PhileKorea Technology, Korea) using $1 \mu \mathrm{l}$ of first-strand cDNA mixed with SYBR Premix Ex TaqTM (Toyobo, Japan). The thermal cycling conditions were $30 \mathrm{~s}$ at $95^{\circ} \mathrm{C}$, followed by 40 cycles of $5 \mathrm{~s}$ at $95^{\circ} \mathrm{C}$ and $31 \mathrm{~s}$ at $58^{\circ} \mathrm{C}$. All $\mathrm{Ct}$ values were collectively analyzed by qbase PLUS version: 2.6.1, Biogazelle, Belgium) and by NormFinder (version 0.953, http://www.multid.se/ genex/hs410.htm) software following published procedures [47,36]; qbasePLUS manual, Biogazelle, Belgium] to compute gene expression stability values (NormFinder).

\section{Availability of supporting data}

The AP2/ERF transcription factors of B. oleracea, B. rapa and $A$. thaliana set supporting the results of this article is available in the public database (http://www.ocri-genomics. org/bolbase/; http://brassicadb.org/brad/; and http:// planttfdb.cbi.edu.cn/, respectively). The micro array data set supporting the result of expression data were downloaded from the AtGenExpress visualization tool (AVT) (http://jsp.weigelworld.org/expviz/expviz.jsp).

\section{Additional files}

\footnotetext{
Additional file 1: Table S1. Complete list of ERF/AP2 transcription factors identified in the cabbage genome from bolbase (http://www. ocri-genomics.org/bolbase/). Table S2. Best BLAST hits of AP2/ERF subfamily domain genes of Chinese cabbage and Arabidopsis used for phylogenetic tree construction. Table S3. Chromosome wise distribution of superfamily genes. Table S4. The paralogous genes of the AP2/ERF superfamily in cabbage. Table S5. B. oleracea AP2/ERF superfamily gene expression profiles in abiotic stresses. The expression
} 
data were downloaded from the AtGenExpress visualization tool (AVT) (http://jsp.weigelworld.org/expviz/expviz.jsp). Table S6. The annotations of all the AP2/ERF genes of cabbage in non-redundant databases from blast2go (www.blast2go.com). Table S7. List of primers used for RT-PCR and real-time PCR analysis.

Additional file 2: Figure S1. Classification of AP2/ERF family transcription factors in cabbage. The size of each segment is proportional to the relative abundance of the assigned AP2/ERF factor. Figure S2. Phylogenetic tree constructed by the neighbor-joining method using AP2 family transcription factor domains in cabbage, Chinese cabbage and Arabidopsis. The numbers are bootstrap values based on 1000 iterations. Figure S3. The DREB subfamily protein motifs derived from each species. Overall the stack indicates the sequence conservation. The height of residues within the stack indicates the relative frequency of each residue at the position. Figure S4. The ERF subfamily protein motifs derived from each species examined. Figure S5. The RAV, AP2 and Soloist family protein motifs derived from each species examined. Figure S6. AP2/ERF protein motifs from each of the species examined. Figure S7. Expression profile cluster analyses of cabbage ERF subfamily genes. Figure S8. Expression profile cluster analyses of cabbage RAV family genes. Figure $\mathbf{S 9}$. Expression profile cluster analyses of cabbage AP2 family genes. Figure S10 (a-e). Identification and characterization of BoCBF genes in B. oleracea, results were revealed based on previous studies $[48,49]$. Detailed description of each figures were described in the ppt section.

\section{Additional file 3: Cold, Salt, drought and ABA.}

\section{Competing interests}

The authors declare that they have no competing interests.

\section{Authors' contributions}

All the authors read and approved the final manuscript. The study was conceived by ISN and JIP. SKTA collected the public dataset of researched and data analysis, bioinformatics analysis, and manuscript preparation. ISN and JIP participated in cabbage and other species planning of analysis and revising the manuscript. HJJ participated in samples collection, data analysis and GPCR validation. ISN and JP participated in data analyses and revised the manuscript.

\section{Acknowledgements}

This research was supported by Golden Seed Project (Center for Horticultural Seed Development, No. 213003- 04-1-CG100), Ministry of Agriculture, Food and Rural Affairs (MAFRA), Ministry of Oceans and Fisheries (MOF), Rural Development Administration (RDA) and Korea Forest Service (KFS).

Received: 9 January 2014 Accepted: 20 May 2014

Published: 3 June 2014

\section{References}

1. Wessler SR: Homing into the origin of the AP2 DNA binding domain. Trends Plant Sci 2005, 10:54-56.

2. Mizoi J, Shinozaki K, Yamaguchi-Shinozaki K: AP2/ERF family transcription factors in plant abiotic stress responses. Biochim Biophys Acta 2011, 1819(2):86-96

3. Licausi F, Giorgi FM, Zenoni S, Osti F, Pezzotti M, Perata P: Genomic and transcriptomic analysis of the AP2/ERF superfamily in Vitis vinifera. BMC Genomics 2010, 11:719.

4. Nakano T, Suzuki K, Fujimura T, Shinshi H: Genome-wide analysis of the ERF gene family in Arabidopsis and rice. Plant Physiol 2006, 140(2):411-432.

5. Hu L, Liu S: AP2/ERF family transcription factors in plant abiotic stress responses. Genet Mol Biol 2011, 34(4):624-633.

6. Sakuma Y, Liu Q, Dubouzet JG, Abe H, Shinozaki K, Yamaguchi-Shinozaki K: DNA-binding specificity of the ERF/AP2 domain of Arabidopsis DREBs, transcription factors involved in dehydration- and cold-inducible gene expression. Biochem Biophys Res Commun 2002, 290(3):998-1009.

7. Li MY, Wang F, Jiang Q, Li R, Ma J, Xiong AS: Genome-wide analysis of the distribution of AP2/ERF transcription factors reveals duplication and elucidates their potential function in Chinese cabbage (Brassica rapa ssp. pekinensis). Plant Mol Biol Rep 2013, 31:1002-1011.
8. Dietz KJ, Vogel MO, Viehhauser A: AP2/EREBP transcription factors are part of gene regulatory networks and integrate metabolic, hormonal and environmental signals in stress acclimation and retrograde signalling. Protoplasma 2010, 245(1-4):3-14.

9. Zhuang J, Cai B, Peng RH, Zhu B, Jin XF, Xue Y, Gao F, Fu XY, Tian YS, Zhao W, Qiao YS, Zhang Z, Xiong AS, Yao QH: Genome-wide analysis of the AP2/ERF gene family in Populus trichocarpa. Biochem Biophys Res Commun 2008, 371(3):468-474.

10. Zhuang J, Chen JM, Yao QH, Xiong F, Sun CC, Zhou XR, Zhang J, Xiong AS Discovery and expression profile analysis of AP2/ERF family genes from Triticum aestivum. Mol Biol Rep 2011, 38(2):745-753.

11. Gil-Humanes J, Piston F, Martin A, Barro F: Comparative genomic analysis and expression of the APETALA2-like genes from barley, wheat, and barley-wheat amphiploids. BMC Plant Biol 2009, 9:66.

12. Zhang G, Chen M, Chen X, Xu Z, Guan S, Li LC, Li A, Guo J, Mao L, Ma Y: Phylogeny, gene structures, and expression patterns of the ERF gene family in soybean (Glycine max L). J Exp Bot 2008, 59(15):4095-4107.

13. $X u Z S$, Chen M, Li LC, Ma YZ: Functions and application of the AP2/ERF transcription factor family in crop improvement. J Integr Plant Biol 2011, 53(7):570-585.

14. Gutterson N, Reuber TL: Regulation of disease resistance pathways by AP2/ERF transcription factors. Curr Opin Plant Biol 2004, 7(4):465-471.

15. Jaglo-Ottosen KR, Gilmour SJ, Zarka DG, Schabenberger O, Thomashow MF: Arabidopsis CBF1 overexpression induces COR genes and enhances freezing tolerance. Science 1998, 280:104-106.

16. Ito Y, Katsura K, Maruyama K, Taji T, Kobayashi M, Seki M, Shinozaki K, Yamaguchi-Shinozaki K: Functional analysis of rice DREB1/CBF-type transcription factors involved in cold-responsive gene expression in transgenic rice. Plant Cell Physiol 2006, 47(1):141-153.

17. Liang $C L$, Li YN, Zhang XP, Song Y, Wang W, Fang J, Cui WM, Jia XD: Immunotoxicologic assessment of genetically modified drought-resistant wheat T349 with GmDREB1. Zhonghua Yu Fang Yi Xue Za Zhi 2012, 46(6):556-560.

18. Hong JP, Kim WT: Isolation and functional characterization of the Ca-DREBLP1gene encoding a dehydration-responsive element binding factor-like protein 1 in hot pepper (Capsicum annuum L. cv. Pukang). Planta 2005, 220(6):875-888.

19. Cheng MC, Liao PM, Kuo WW, Lin TP: The Arabidopsis ethylene response factor 1 regulates abiotic stress-responsive gene expression by binding to different cis-acting elements in response to different stress signals. Plant Physiol 2013, 162:1566-1582.

20. Fujita Y, Fujita M, Shinozaki K, Yamaguchi-Shinozaki K: ABA-mediated transcriptional regulation in response to osmotic stress in plants. J Plant Res 2011, 124(4):509-525.

21. Mun JH, Kwon SJ, Yang TJ, Seol YJ, Jin M, Kim JA, Lim MH, Kim JS, Baek S, Choi BS, Yu HJ, Kim DS, Kim N, Lim KB, Lee SI, Hahn JH, Lim YP, Bancroft I, Park BS: Genome-wide comparative analysis of the Brassica rapa gene space reveals genome shrinkage and differential loss of duplicated genes after whole genome triplication. Genome Biol 2009, 10:R111.

22. Yu J, Zhao M, Wang X, Tong C, Huang S, Tehrim S, Liu Y, Hua W, Liu S: Bolbase: a comprehensive genomics database for Brassica oleracea. BMC Genomics 2013, 14:664.

23. Song X, Li Y, Hou X: Genome-wide analysis of the AP2/ERF transcription factor superfamily in Chinese cabbage (Brassica rapa ssp. pekinensis). BMC Genomics 2013, 14:573.

24. Kilian J, Whitehead D, Horak J, Wanke D, Weinl S, Batistic O, D'Angelo C, Bornberg-Bauer E, Kudla J, Harter K: The AtGenExpress global stress expression data set: protocols, evaluation and model data analysis of UV-B light, drought and cold stress responses. Plant J 2007, 50:347-363.

25. Liu Z, Kong L, Zhang M, Lv Y, Liu Y, Zou M, Lu G, Cao J, Yu X: Genome-wide identification, phylogeny, evolution and expression patterns of ap2/erf genes and cytokinin response factors in Brassica rapa ssp. Pekinensis. PLoS One 2013, 8(12):e83444.

26. Medina J, Bargues M, Terol J, Perez-Alonso M, Salinas J: The Arabidopsis CBF gene family is composed of three genes encoding AP2 domain-containing proteins whose expression is regulated by low temperature but not by abscisic acid or dehydration. Plant Physiol 1999, 119:463-469.

27. Szklarczyk D, Franceschini A, Kuhn M, Simonovic M, Roth A, Minguez $P$, Doerks T, Stark M, Muller J, Bork P, Jensen LJ, von Mering C: The STRING database in 2011: functional interaction networks of proteins, 
globally integrated and scored. Nucleic Acids Res 2011, 39(Database issue):D561-D568.

28. Thomashow MF: Role of cold-responsive genes in plant freezing tolerance. Plant Physiol 1998, 118:1-7.

29. Stockinger EJ, Gilmour SJ, Thomashow MF: Arabidopsis thaliana CBF1 encodes an AP2 domain-containing transcriptional activator that binds to the C-repeat/DRE a cis-acting DNA regulatory element that stimulates transcription in response to low temperature and water deficit. Proc Natl Acad Sci U S A 1997, 94:1035-1040.

30. Qin F, Kakimoto M, Sakuma Y, Maruyama K, Osakabe Y, Tran LS, Shinozaki K, Yamaguchi-Shinozaki K: Regulation and functional analysis of ZmDREB2A in response to drought and heat stresses in Zea mays L. Plant J 2007, 50(1):54-69.

31. Kidokoro S, Maruyama K, Nakashima K, Imura Y, Narusaka Y, Shinwari ZK, Osakabe Y, Fujita Y, Mizoi J, Shinozaki K, Yamaguchi-Shinozaki K: The phytochrome-interacting factor PIF7 negatively regulates DREB1 expression under circadian control in Arabidopsis. Plant Physiol 2009, 151(4):2046-2057.

32. Hark AT, Vlachonasios KE, Pavangadkar KA, Rao S, Gordon H, Adamakis ID, Kaldis A, Thomashow MF, Triezenberg SJ: Two Arabidopsis orthologs of the transcriptional coactivator ADA2 have distinct biological functions. Biochim Biophys Acta 2009, 1789(2):117-124.

33. Haake V, Cook D, Riechmann JL, Pineda O, Thomashow MF, Zhang JZ: Transcription factor CBF4 is a regulator of drought adaptation in Arabidopsis. Plant Physiol 2002, 130(2):639-648.

34. Hong SW, Jon JH, Kwak JM, Nam HG: Identification of a receptor-like protein kinase gene rapidly induced by abscisic acid, dehydration, high salt, and cold treatments in Arabidopsis thaliana. Plant Physiol 1997, 4:1203-1212.

35. Kirch HH, van Berkel J, Glaczinski H, Salamini F, Gebhardt C: Structural organization, expression and promoter activity of a cold-stress-inducible gene of potato. Plant Mol Biol 1997, 5:897-909.

36. Andersen $C L$, Jensen $J L$, Orntoft TF: Normalization of real-time quantitative reverse transcription-PCR data: a model-based variance estimation approach to identify genes suited for normalization, applied to bladder and colon cancer data sets. Cancer Res 2004, 64:5245-5250.

37. Wang X, Wang H, Wang J, Sun R, Wu J, Liu S, Bai Y, Mun JH, Bancroft I, Cheng F, Huang S, Li X, Hua W, Wang J, Wang X, Freeling M, Pires JC, Paterson AH, Chalhoub B, Wang B, Hayward A, Sharpe AG, Park BS, Weisshaar B, Liu B, Li B, Liu B, Tong C, Song C, Duran C, et al: The genome of the mesopolyploid crop species Brassica rapa. Nat Genet 2011, 43(10):1035-1039.

38. Cheng F, Liu S, Wu J, Fang L, Sun S, Liu B, Li P, Hua W, Wang X: BRAD, the genetics and genomics database for Brassica plants. BMC Plant Biol 2011 11:136.

39. Zhang H, Jin J, Tang L, Zhao Y, Gu X, Gao G, Luo J: PlantTFDB 2.0: update and improvement of the comprehensive plant transcription factor database. Nucleic Acids Res 2011, 39(Database issue):D1114-D1117.

40. Jin J, Zhang H, Kong L, Gao G, Luo J: PlantTFDB 3.0: a portal for the functional and evolutionary study of plant transcription factors. Nucleic Acids Res 2013, 42:1-6.

41. Letunic I, Doerks T, Bork P: SMART 7: recent updates to the protein domain annotation resource. Nucleic Acids Res 2012, 40(Database issue):D302-D305.

42. Bailey TL, Boden M, Buske FA, Frith M, Grant CE, Clementi L, Ren J, Li WW, Noble WS: MEME SUITE: tools for motif discovery and searching. Nucleic Acids Res 2009, 37(Web Server issue):W202-W208.

43. Voorrips RE: MapChart: software for the graphical presentation of linkage maps and QTLs. J Hered 2002, 93(1):77-78.

44. Tamura K, Peterson D, Peterson N, Stecher G, Nei M, Kumar S: MEGA5: molecular evolutionary genetics analysis using maximum likelihood, evolutionary distance, and maximum parsimony methods. Mol Biol Evol 2011, 28(10):2731-2739.

45. Conesa A, Gotz S, Gomez JMG, Terol J, Talon M, Robles M: Blast2GO: a universal tool for annotation, visualization and analysis in functional genomics research. Bioinformatics 2005, 21(18):3674-3676.

46. Thamil Arasan SK, Park Jl, Ahmed NU, Jung HJ, Lee IH, Cho YG, Lim YP Kang KK, Nou IS: Gene ontology based characterization of Expressed Sequence Tags (ESTs) of Brassica rapa cv Osome. Indian J Exp Biol 2013, 51:530.
47. Hellemans J, Mortier G, Paepe A, Speleman F, Vandesompele J: qBase relative quantification framework and software for management and automated analysis of real-time quantitative PCR data. Genome Biol 2007, 8:R19.

48. Buttner M, Singh KB: Arabidopsis thaliana ethylene responsive element binding protein (AtEBP), an ethyleneinducible, GCC box DNA-binding protein interacts with an ocs element binding protein. Proc Natl Acad SCl U S A 1997, 94:5961-5966.

49. Okamuro JK, Caster B, Villarroel R, Van Montagu M, Jofuku DK: The AP2 domain of APETALA2 defines a large new family of DNA binding proteins in Arabidopsis. Proc Natl Acad Sci U S A 1997, 13:7076-7081.

doi:10.1186/1471-2164-15-422

Cite this article as: Thamilarasan et al:: Genome-wide analysis of the distribution of AP2/ERF transcription factors reveals duplication and CBFs genes elucidate their potential function in Brassica oleracea. BMC Genomics 2014 15:422.

\section{Submit your next manuscript to BioMed Central and take full advantage of:}

- Convenient online submission

- Thorough peer review

- No space constraints or color figure charges

- Immediate publication on acceptance

- Inclusion in PubMed, CAS, Scopus and Google Scholar

- Research which is freely available for redistribution 